\title{
Pseudoaneurisma de arteria renal principal asociado a fistula cecal tras nefrectomia radical: caso clínico y revisión de la literatura
}

\author{
Hernando Arteche A. ${ }^{1 a}$, Alpuente Roman C. ${ }^{1 a}$, Martin del Toro R... ${ }^{2 b}$, Pérez-Piqueras Gómez A. ${ }^{3 c}$, \\ Sainz González F.2c, Cabrera Cabrera JA.2a
}

Sanid. mil. 2014; 70 (2): 95-97; ISSN: 1887-8571

\begin{abstract}
RESUMEN
Las complicaciones vasculares secundarias al tratamiento quirúrgico del cáncer de riñón localizado son más frecuentes cuando se realiza una cirugía conservadora de nefronas. Sin embargo, la nefrectomía radical no está exenta de ellas. Presentamos el caso de un pseudoaneurisma de la arteria renal principal con comunicación fistulosa a colon ascendente tras nefrectomía radical. El paciente debutó con un episodio de shock hipovolémico debido a hemorragia digestiva baja.
\end{abstract}

PALABRAS CLAVE: Pseudoaneurisma arteria renal, fístula arterioentérica, hemorragia digestiva baja, nefrectomía radical.

Pseudoaneurysm of the main renal artery associated to cecal fistula after radical nephrectomy: a clinical case and literature review SUMMARY: Vascular complications secondary to surgical treatment of localized kidney cancer are more frequent when a nephronsparing surgery is performed. However, radical nephrectomy is not exempt from them. We report the case of a pseudoaneurysm of the main renal artery with fistulous communication to ascending colon after radical nephrectomy. The patient presented with an episode of hypovolemic shock due to lower gastrointestinal bleeding.

KEY WORDS: Renal artery pseudoaneurysm, arterial-enteric fistula, lower gastrointestinal bleeding, radical nephrectomy.

\section{CASO CLINICO}

Mujer de 75 años de edad diagnosticada de un tumor de $7 \mathrm{~cm}$ dependiente del polo inferior del riñón derecho en el seno del estudio de una hematuria asintomática. El tumor infiltraba la pelvis renal y se extendía hacia la vena renal derecha sin trombosis tumoral en vena cava inferior. Se procedió a nefrectomía radical derecha laparoscópica que precisó convertir a cirugía abierta por sangrado de vena gonadal en su inserción en vena cava inferior. El examen anatomopatológico reveló un carcinoma renal de células claras, Furhman 3, pT3 Nx, con márgenes quirúrgicos negativos. El postoperatorio transcurrió sin incidencias siendo dada de alta al décimo día postoperatorio.

Al decimoquinto día de la cirugía la paciente presentó cuadro de dolor abdominal focalizado en flanco derecho y síncope en su domicilio. En la analítica presentaba anemización significativa (Hemoglobina de $8,4 \mathrm{~g} / \mathrm{dl}$ y hematocrito de $25,4 \%$ ) y leucocitosis de 27.760 con $85 \%$ de neutrófilos. La TAC abdominopélvica reveló un hematoma retroperitoneal de $10 \mathrm{~cm}$ de diámetro en lecho quirúrgico que requirió exploración quirúrgica evacuando dicho hematoma y realizando hemostasia de sangrado difuso, sin encontrar punto de sangrado

${ }^{1}$ Facultativo Especialista de Área.

${ }^{2}$ Tcol. Médico.

${ }^{3}$ Cte. Médico.

a Servicio de Urología.

b Servicio de Cirugía General.

c Servicio de Cirugía Vascular.

Hospital Central de la Defensa Gómez Ulla. Madrid. España.

Dirección para correspondencia: Alberto Hernando Arteche. Servicio de Urología, Hospital Central de la Defensa "Gómez Ulla". Glorieta del Ejército 1, 28047. Madrid, España. 91422 2412. albherart@gmail.com

Recibido: 10 de julio de 2013

Aceptado: 2 de diciembre de 2013 activo. La recuperación de la paciente fue satisfactoria presentando buena tolerancia oral y recuperación del tránsito intestinal.

A los 17 días presentó cuadro agudo de hemorragia digestiva baja con shock hipovolémico refractario a sueroterapia siendo preciso ingreso en UCI. En la analítica sanguínea presentaba descenso de hematocrito de $34.5 \%$ a $25.9 \%$. Se realizó endoscopia digestiva baja urgente identificando estenosis de la luz colónica a nivel de ángulo hepático secundaria a compresión extrínseca sin poder sobrepasar; y en el TAC abdominal un hematoma en lecho de nefrectomía derecha y una masa retrocava de $33 \mathrm{x}$ 25 x $43 \mathrm{~mm}$ compatible con pseudoaneurisma de la arteria renal principal derecha con comunicación fistulosa a colon derecho (Fig. 1-3). Se procedió a intervención quirúrgica urgente localizando el origen de la arteria renal derecha a nivel del ángulo de Treitz y realizando ligadura de la misma. De esa manera, se interrumpió el flujo sanguíneo hacia el pseudoaneurisma y la fístula digestiva. Se completó la intervención evacuando el hematoma en lecho de nefrectomía y realizando hemicolectomía derecha.

\section{DISCUSION}

Las lesiones aneurismáticas son dilataciones anormales de la luz de los vasos secundarias a una enfermedad o agresión en la pared de los mismos. Estas lesiones se clasifican en dos tipos según la composición de su pared: aneurismas verdaderos y pseudoaneurismas. Los aneurismas verdaderos son dilataciones de la luz arterial con preservación de las 3 capas del vaso (intima, media y adventicia) ${ }^{1}$. Sin embargo, los pseudoanerismas se producen como resultado de la lesión de una o más capas de la pared arterial. La compresión del tejido periarterial hace que el sangrado sea lento y forme un hematoma alrededor de la arte- 


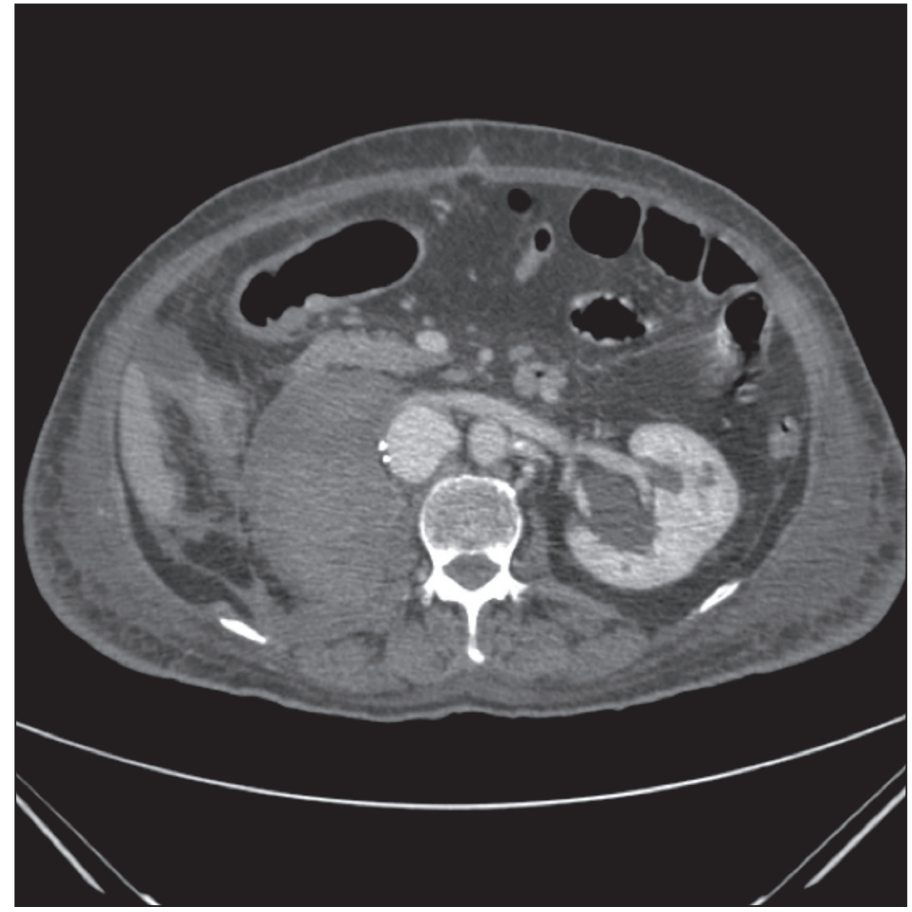

Figura 1. TAC mostrando masa retrocava contrastada de $3 \mathrm{~cm}$ en continuidad con arteria renal derecha correspondiente a pseudoaneurisma. La vena cava se encuentra colapsada formando una imagen de semiluna.

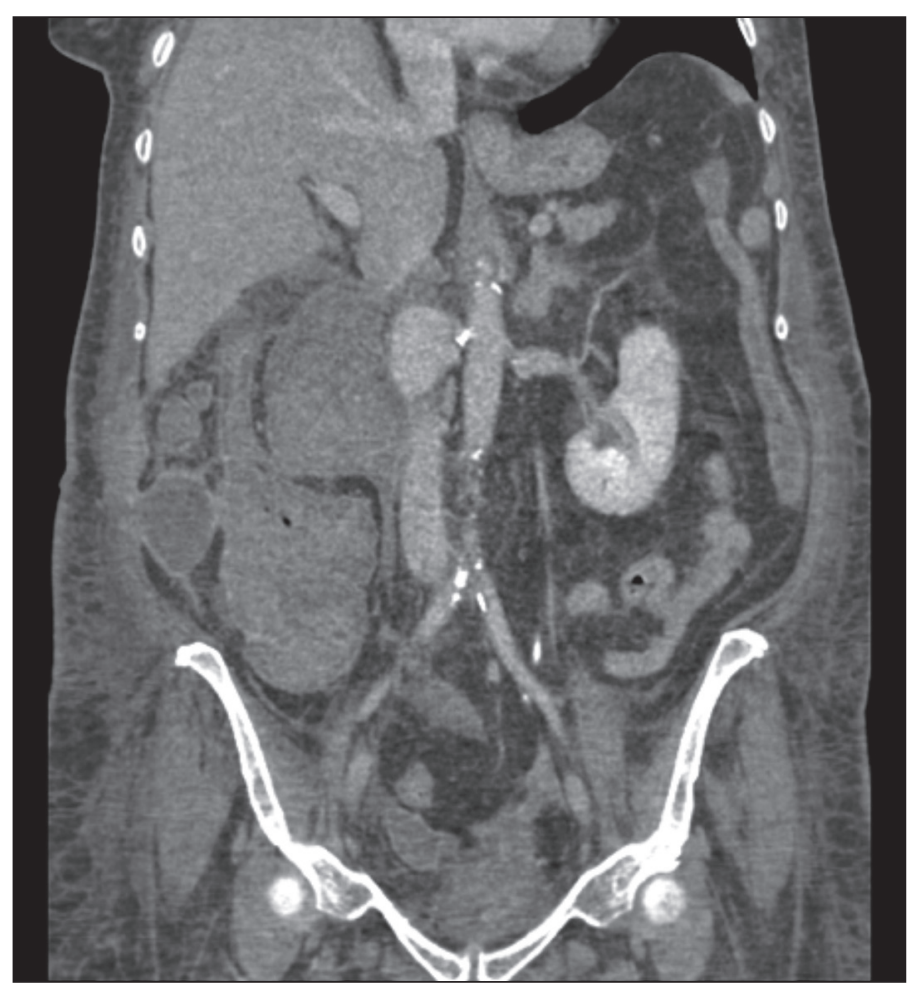

Figura 2. Reconstrucción coronal de TC mostrando pseudoaneurisma procedente de la arteria renal derecha con calcificación en su osium.

riotomía. A continuación, se produce una reacción fibrosa que encapsula la hemorragia formando una estructura que asemeja un saco aneurismático ${ }^{2}$.

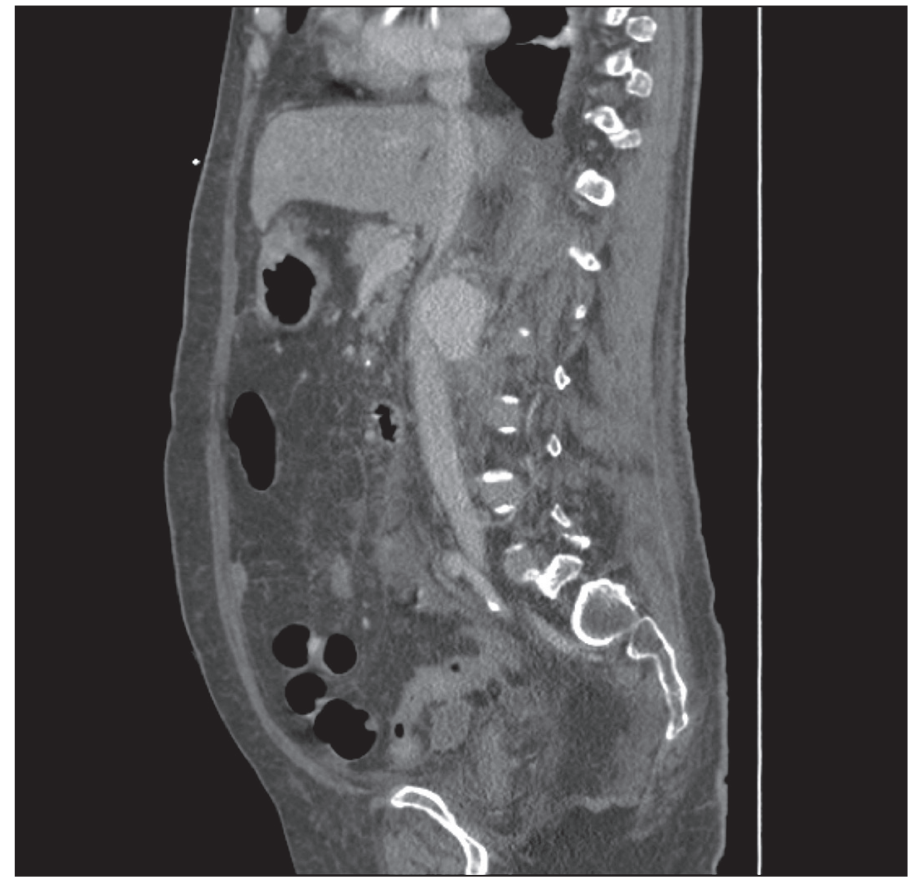

Figura 3. Reconstrucción sagital de TC mostrando colapso y desplazamiento anterior de la vena cava secundario a pseudoaneurisma.

En nuestro caso, la hipótesis planteada fue que el hematoma del lecho de la nefrectomía fue producido por un sangrado secundario a una mala oclusión arterial previa a la formación del pseudoaneurisma. En la TAC previa a la intervención no se objetivaba fuga de contraste que hiciese sospechar el origen del sangrado ni la presencia de saco aneurismático a nivel de la arteria renal principal derecha.

El desarrollo de un hematoma del lecho quirúrgico tras nefrectomía radical es poco frecuente y normalmente se debe a una lesión de pequeños vasos lumbares o sangrado lento procedente del tejido pararrenal. Solo cuando se produce una anemización brusca con evidencia de sangrado activo, se trata de un gran hematoma o se produce una sobreinfección del mismo es obligado la revisión quirúrgica. La incidencia real de hematomas de escaso volumen o asintomáticos es desconocida dado que, normalmente, no se realizan pruebas de imagen en el postoperatorio próximo a la nefrectomía si la evolución es satisfactoria.

Posteriormente, en nuestro caso, tras una evolución satisfactoria, se produjo un shock hipovolémico secundario a sangrado digestivo bajo. Cuando realizamos la TAC correspondiente se diagnosticó un hematoma similar al previo, aunque esta vez sí existía evidencia de la formación de un pseudoaneurisma a nivel de la ligadura de la arteria renal principal derecha. Y por otro lado, la presencia de una comunicación fistulosa entre el hematoma y la pared del ciego fue identificada como la causa del sangrado digestivo bajo.

Aunque se trata de una hipótesis, pensamos que el hematoma formado, acompañado de una reacción fibrosa, erosionó la pared intestinal que hasta ese momento era la contención del trayecto fistuloso procedente del pseudoaneurisma. Otra explicación podría ser la presencia de una lesión inadvertida en la pared intestinal, aunque nos parece menos probable dada la ausencia de sintomatología abdominal que hiciera pensar en una fístula entérica, y la ausencia de contenido intestinal intraperito- 


\section{Pseudoaneurisma de arteria renal principal asociado a fistula cecal tras nefrectomia radical: caso...}

neal durante la reintervención. A ello se suma el hecho de que la paciente presentaba tolerancia oral y tránsito intestinal satisfactorio hasta el desarrollo del cuadro agudo.

La formación de pseudoaneurismas en la arteria renal principal tras una nefrectomía radical es poco frecuente. Revisando la literatura, hemos encontrado un caso descrito en un paciente tras nefrectomía de donante vivo ${ }^{3}$ y otro en nefrectomía radical por tumor renal ${ }^{4}$. Más frecuentes son aquellos producidos tras una nefrectomía parcial, que alcanzan una incidencia de 1,2 a 2,3\% Estos tienen su origen en arterias intrarrenales como consecuencia de la exéresis del parénquima y la presencia de un fluJo hemorrágico lento que condiciona la formación de un pseudoaneurisma.

También se han descrito pseudoaneurismas secundarios a angioplastia y colocación de stent por estenosis de la arteria renal ${ }^{5} \mathrm{y}$ pseudoaneurismas en la arteria renal principal de un riñón trasplantado, normalmente resultado de una infección del injerto o fallo técnico de la anastomosis vascular ${ }^{2,6}$. Otros casos de pseudoaneurismas son los debidos a procedimientos percutáneos o endourologicos (biopsia percutánea, nefrostomía o nefrolitotomía, ureteroscopia terapéutica y endopielotomía), trauma renal y enfermedades sistémicas como vasculitis (Enfermedad de Beçhet) y linfomas².

El tratamiento de esta grave complicación no está consensuado debido a la escasa frecuencia de presentación. Ngo et al publican un algoritmo de tratamiento en una revisión de pseudoaneurismas renales ${ }^{2}$. El seguimiento de estas lesiones estaría indicado en un grupo seleccionado de pacientes cuando no existe complicación del mismo o si no existe otro tratamiento posible. Sin embargo, cuando se trata de un pseudoaneurisma complicado, bien por hemorragia, infección o fístula hemato-entérica como en nuestro caso, el tratamiento quirúrgico es obligado. La embolización percutánea es un procedimiento menos invasivo y con una alta efectividad en casos de pseudoaneurismas de pequeños vasos renales. Sin embargo, cuando existe inestabilidad hemodinámica por sangrado de pseudoaneurisma, la embolización percutánea solo debe hacerse cuando esté disponible un equipo de radiología intervencionista cualificado sin un retraso sustancial en obtener una angiografía del paciente ${ }^{7-9}$.

Las fistulas arterioentéricas representan una rara complicación que requiere intervención inmediata. Se definen como una comunicación entre un vaso arterial mayor y el sistema digestivo. Las causas de fistulas arterioentéricas primarias incluyen aneurismas, procesos infecciosos, ulceras pépticas, traumatismos, enfermedad inflamatoria intestinal, neoplasias y radioterapia. Las fístulas secundarias, habitualmente, son debidas a complicaciones tardías de cirugías abdominales paraaórticas y reparación de aneurismas aórticos ${ }^{10}$. La patogénesis del proceso de fistulización tiene diferentes teorías: infección local, lesión directa intestinal en procedimientos quirúrgicos y erosión de la pared intestinal por pulsos traumáticos repetitivos ${ }^{11}$. En nuestro caso, la teoría más plausible corresponde al desarrollo de una reacción fibrosa y erosión del hematoma sobre la pared intestinal.

Clínicamente, el sangrado gastrointestinal o la sintomatología infecciosa son las dos formas de presentación más frecuentes de un proceso de fistulización arterioentérica. En la mayoría de los casos, el sangrado inicial se interrumpe debido a la oclusión de la pequeña comunicación fistulosa. Esta detención, nos permite obtener tiempo para una rápida evaluación y establecimiento del diagnóstico correcto, ya que un sangrado subsecuente podría deparar consecuencias fatales ${ }^{12}$. El tratamiento más aceptado consiste en la cirugía abierta. Sin embargo, un manejo arterial endovascular podría reducir las tasas de morbilidad y mortalidad perioperatoria, y estabilizar al paciente si una intervención quirúrgica posterior fuera necesaria ${ }^{10}$. Se trataría de un procedimiento "puente" hacia la cirugía abierta cuando se hace factible el empleo de dicha técnica ${ }^{13}$.

\section{CONCLUSIONES}

La presencia de una hemorragia súbita y diferida tras nefrectomía radical es poco frecuente y su presencia debe hacernos sospechar, entre otras causas, la ruptura de un pseudoaneurisma de la arteria renal principal. Menos frecuente es el desarrollo de una fístula arterioentérica concomitante.

El tratamiento de esta grave complicación debe ser urgente mediante embolización endovascular (si existe disponibilidad de un equipo de radiología intervencionista cualificado) o mediante cirugía urgente realizando el cierre de la arteriotomía o ligadura de la porción arterial proximal al pseudoaneurisma, como presentamos en nuestro caso.

\section{BIBLIOGRAFÍA}

1. Cura M, Elmerhi F, Bugnogne A, Palacios R, Suri R, Dalsaso T. Renal aneurysms and pseudoaneurysms. Clin Imaging. $2011 \mathrm{Jan}-\mathrm{Feb}$; 35(1):29-41.

2. Ngo TC, Lee JJ, Gonzalgo ML. Renal pseudoaneurysm: an overview. Nat Rev Urol. 2010 Nov; 7(11):619-25.

3. Simforoosh N, Sarhangnejad R, Basiri A, Ziaee SA, Sharifiaghdas F, Tabibi A, Nouralizadeh A, Kashi AH, Moosanejad N. Vascular Clips Are Safe and a Great Cost-Effective Technique for Arterial and Venous Control in Laparoscopic Nephrectomy: Single-Center Experience with 1834 Laparoscopic Nephrectomies. J Endourol. 2012 Apr 16

4. Bozgeyik Z, Ozdemir H, Orhan I, Cihangiroglu M, Cetinkaya Z. Pseudoaneurysm and renal arteriovenous fistula after nephrectomy: two cases treated by transcatheter coil embolization. Emerg Radiol. 2008 Mar; 15(2):119-22.

5. Deitch JS, Hansen KJ, Regan JD, Burkhart JM, Ligush J Jr. Infected renal artery pseudoaneurysm and mycotic aortic aneurysm after percutaneous transluminal renal artery angioplasty and stent placement in a patient with a solitary kidney. J Vasc Surg. 1998 Aug; 28(2):340-4.

6. Al-Wahaibi KN, Aquil S, Al-Sukaiti R, Al-Riyami D, Al-Busaidi Q. Transplant renal artery false aneurysm: case report and literature review. Oman Med J. 2010 Oct; 25(4):306-10.

7. Poulakis V, Ferakis N, Becht E, Deliveliotis C, Duex M. Treatment of renal-vascular injury by transcatheter embolization: immediate and long-term effects on renal function. J Endourol. 2006 Jun; 20(6):405-9.

8. Ghoneim TP, Thornton RH, Solomon SB, Adamy A, Favaretto RL, Russo P. Selective arterial embolization for pseudoaneurysms and arteriovenous fistula of renal artery branches following partial nephrectomy. J Urol. 2011 Jun; 185(6):2061-5

9. Sildiroglu O, Saad WE, Hagspiel KD, Matsumoto AH, Turba UC. Endovascular Management of Iatrogenic Native Renal Arterial Pseudoaneurysms. Cardiovasc Intervent Radiol. 2011 Dec 15.

10. Leonhardt H, Mellander S, Snygg J, Lönn L. Endovascular management of acute bleeding arterioenteric fistulas. Cardiovasc Intervent Radiol. 2008 May-Jun; 31(3):542-9.

11. Bergqvist D, Björck M. Secondary arterioenteric fistulation--a systematic literature analysis. Eur J Vasc Endovasc Surg. 2009 Jan; 37(1):31-42.

12. Baird RL, Slagle GW, Boggs HW Jr. Arterio-enteric fistulas. Dis Colon Rectum. 1979 Apr; 22(3):187-8.

13. Kakkos SK, Papadoulas S, Tsolakis IA. Endovascular management of arterioenteric fistulas: a systemic review and meta-analysis of the literature. J Endovasc Ther. 2011 Feb; 18(1):66-77. 